\title{
The role of PDL-cementum enthesis in protecting PDL under masticatory loading: A finite element investigation
}

Hossein Jokar

Amirkabir University of Technology Department of Biomedical Engineering

Gholamreza Rouhi ( $\square$ grouhi@aut.ac.ir)

Amirkabir University of Technology Department of Biomedical Engineering https://orcid.org/00000001-5592-4970

\section{Nabiollah Abolfathi}

Amirkabir University of Technology Department of Biomedical Engineering

\section{Research Article}

Keywords: PDL-cementum enthesis, Periodontal ligament, Tooth-periodontium-bone complex, Strain energy density, Finite element analysis

Posted Date: April 16th, 2021

DOI: https://doi.org/10.21203/rs.3.rs-315785/v1

License: (c) (i) This work is licensed under a Creative Commons Attribution 4.0 International License. Read Full License 


\section{Abstract}

PURPOSE. Function of periodontal ligament-cementum enthesis (PCE) in transferring mechanical stimuli within tooth-periodontium (PDT)-bone complex was not made clear yet. This study aimed to evaluate the effects of PCE on the mechanical stimuli distribution within the PDL and alveolar bone in the tooth-PDTbone complex under occlusal forces using finite element method (FEM).

METHODS. A computed tomography (CT) based model of alveolar bone and $2^{\text {nd }}$ premolar of mandible was constructed, in which the PDT was considered at the interface of alveolar bone and tooth. Under a 3MPa distributed occluso-apical masticatory load, applied over the uppermost surface of crown, the von Mises strain (VMST) and strain energy density (SED) within PDL, and von Mises stress (VMSR) and SED within alveolar bone were calculated in two situations: 1 . When the PCE was absent; and 2. When the PCE was present between the PDL and cementum.

RESULTS. PCE levels-off the SED and vMST within PDL by maximum values of $92 \mathrm{kPa}$ and 0.04 $\mathrm{mm} / \mathrm{mm}$, respectively, compared to the model without PCE. Moreover, it increased the alveolar bone SEDs and VMSR by maximum values of $0.36 \mathrm{kPa}$ and $0.63 \mathrm{MPa}$, respectively, compared to the without PCE model.

CONCLUSION. By including PCE in the tooth-PDT-bone model, the mechanical stimuli shift from PDL to its surrounding alveolar bone. Thus, it can be speculated that the tooth-PDT-bone complex has the capability of, through shifting excess mechanical stimuli from PDL toward the alveolar bone, reducing the risk of PDL damage.

\section{Introduction}

In load bearing biological systems, the physical properties of tissues and their interfaces have significant impact on their biomechanical functions [1]. In the musculoskeletal system, soft to hard tissues attachment sites, so-called entheses, including ligament-bone, and tendon-bone interfaces, are smartly designed, which transfer loads in a way to reduce the probability of failure and also to facilitate relative motion between the soft and hard tissues [2]. From the biomechanical perspective, the entheses are exemplary load transmitter, which experience modeling and remodeling processes at a higher rate than their adjacent tissues [3, 4]. Gradual changes in mechanical properties develop within entheses, as an adaptive response in order to strengthen their neighboring tissues and take proper care of the externally applied loads [5].

There are four regions at the interface of alveolar bone-tooth construct, including: (1) PDL region; (2) PDLcementum enthesis; (3) bulk cementum; and (4) cementum-dentin junction (CDJ) [1]. Ho and colleagues showed that the collagen fibers are continuously distributed all the way from the PDL, through PCE, bulk cementum, and CDJ [1]. They also found that PCE and CDJ have similar compositions, and their nanomechanical properties are similar and are in the same range [1]. Lin and co-workers hypothesized that entheses prevent the risk of irreversible failure during prolonged cyclic loading [5]. The failure 
prevention mechanism of enthesis requires that it reduces the transferred mechanical stimuli from hard to soft tissues, and avoids stress concentration, and thus the construct acts similar to a functionally graded material. To date, finite element method (FEM) was employed by many researchers, as a powerful tool to discover new aspects of the problems related to the fields of dental and/or orthopedic biomechanics [6-9]. Ren and co-workers in a finite element study found that cementum and CDJ have a significant impact on reducing PDL strains during mastication [10]. Nikolaus and co-workers investigated the effect of considering variable thickness for PDL on the mechanical stimuli distribution within toothPDT-bone complex. Their FE study showed that by considering variable thickness for PDL, there would be an increase in bone stress, while the PDL experiences lower strain [11]. They concluded that the toothPDT-bone complex has the capability of reducing the risk of failure of PDL by transferring the excess loads from PDL towards the alveolar bone.

Despite recent research related to the PCE, enough attention was not paid to its function in mechanical stimuli transmission within tooth-PDT-bone construct, especially from cementum to PDL, and from PDL to alveolar bone. Based on the previous studies, one of the hypothesized functions considered for entheses is to reduce the experienced mechanical stimuli within the soft tissues, and consequently prevent their permanent damages [5]. The main purpose of this study was to evaluate the effects of PCE on the mechanical stimuli transmission from cementum to PLD, and also from PDL to alveolar bone, using finite element method. Thus, the displacement of tooth, the VMST and SED within PDL, and vMSR and SED within alveolar bone of a tooth-PDT-bone complex were found under masticatory loads, using FEM.

\section{Materials And Methods}

\subsection{Construction of the CAD Models}

CT data was used to create CAD models of the 2 nd premolar, tooth No. 20, and mandible (Fig. 1). The participant was a 20-year-old healthy male and provided the informed consent, which was approved by the Institutional Review Board of Tehran University of Medical Science. Mimics (V10.01, Materialise, Leuven, Belgium) was used for obtaining the point clouds, and the models of the tooth and mandible were constructed by Catia (V5.R19, Dassault Systems, Paris, France). Mandible consisted a cancellous core with an outer compact shell, and periodontium tissue, dentin and enamel were the components of the tooth. Periodontium tissue consisted four distinct layers including: PDL, PCE, cementum, and CDJ (Fig. 1) [1]. The uniform thicknesses of $200 \mu \mathrm{m}, 20 \mu \mathrm{m}, 150 \mu \mathrm{m}$ and $50 \mu \mathrm{m}$ were assigned to the PDL, PCE, cementum and CDJ, respectively $[12,1,13-15]$.

In this study, two models were created as follows. The 1st model did not include PCE, i.e. without PCE model (woPCE), which consisted a 2nd premolar tooth and the alveolar bone. In this model, the periodontium tissue was at the interface of the tooth and mandible (Fig. 1d). The second model includes PCE, i.e. with PCE model (WPCE), which was similar to the first one, but the PCE as a thin layer with a thickness of $20 \mu \mathrm{m}$ was considered between PDL and cementum (Fig. 1e). 


\subsection{Material Properties}

To simulate the large deformation of the PDL under occlusal forces [12], a nonlinear elastic model was employed in this study, which was extracted from a quasi-static response of the PDL [16]. For other tissues, homogenous, isotropic, and linear elastic materials were considered (Table 1).

Table 1

Material properties assigned to the components of woPCE and wPCE models

\begin{tabular}{|lll|}
\hline Materials & Young's modulus (MPa) & Poisson's ratio \\
\hline Spongy Bone [17] & 1,370 & 0.3 \\
\hline Cortical Bone [17] & 13,700 & 0.3 \\
\hline PDL-cementum enthesis [1, 7] & 2,000 & 0.35 \\
\hline Cementum [18, 12] & 6,800 & 0.31 \\
\hline Cementum-dentin junction [18, 12, 1] & 2,990 & 0.35 \\
\hline Dentin [17] & 18,600 & 0.31 \\
\hline Enamel [17] & 41,000 & 0.3 \\
\hline
\end{tabular}

\subsection{Finite Element Model Generation and Analysis}

Finite element analysis was performed using ANSYS (ANSYS Inc., Canonsburg, Pennsylvania, United States). The wOPCE and wPCE FE models include 331,540 and 333,138 tetrahedral solid structural elements, respectively. For all models, the mesh convergence was successfully checked by reducing the mesh sizes until the further refinement of the elements' sizes did not cause alteration in the maximum values of PDL VMST by more than $5 \%$.

In the Cartesian coordinate system for the finite element models, the X-axis coincides with the mesiodistal, the Y-axis with bucco-lingual, and the Z-axis with occluso-apical orientations of the tooth (Fig. 1). In the alveolar bone, the distal and mesial faces were constrained in all directions (Fig. 1f). The contact faces in all interfaces of both models were assumed to be bonded $[19,20]$. These connections include the interfaces between layers of PDT, i.e. PDL to PCE, PCE to cementum, and cementum to CDJ, and the interfaces between other connected tissues, i.e. CDJ to root dentin, mandible to the PDL, and cancellous bone to compact bone.

The maximum measured force on the human tooth was about $700 \mathrm{~N}[21,22]$, but usually each tooth tolerates $100 \mathrm{~N}$ occluso-apical force during mastication [23,24]. Thus, in this study, a $3 \mathrm{MPa}$ distributed masticatory load, equivalent to a $100 \mathrm{~N}$ concentrated occluso-apical force, was applied to the $2 \mathrm{nd}$ premolar tooth on the uppermost surface of the crown, with a total area of $33.4 \mathrm{~mm}^{2}$ (Fig. 1f). The movement pattern of tooth under masticatory force, VMST and SED of PDL, and VMSR and SED of alveolar bone were then calculated in woPCE and wPCE FE models. 


\subsection{Validation of finite element model}

Results of the finite element model regarding the displacement-force pattern of the 2nd premolar tooth in the WPCE model, under distributed masticatory load, were compared (Fig. 2) with the displacement-force patterns of teeth reported in Brunski's experimental study [25], and finite element studies of Maceri et al. [26], and Ren et al. [10]. The maximum, and mean difference, between displacement of the 2nd premolar tooth in the wPCE model, and displacement of teeth reported in the above-mentioned studies $[26,10,25]$, under the same masticatory loading condition, and their Pearson value can be found in Table 2.

Table 2

The maximum, and mean difference, between displacement of the 2 nd premolar tooth in the WPCE model and displacement of teeth reported in some other studies [26, 10,25], under the same masticatory loading condition, and their Pearson values.

\begin{tabular}{|llllc|}
\hline Study & Tooth & $\begin{array}{l}\text { Max. Difference in } \\
\text { displacement }(\mu \mathrm{m})\end{array}$ & $\begin{array}{l}\text { Mean Difference in } \\
\text { displacement }(\mu \mathrm{m})\end{array}$ & $\begin{array}{l}\text { P- } \\
\text { Value }\end{array}$ \\
\hline $\begin{array}{l}\text { Brunski } \\
{[25]}\end{array}$ & $\begin{array}{l}\text { 1st Maxillary } \\
\text { Molar }\end{array}$ & 7.24 & 4.91 & 0.96 \\
\hline $\begin{array}{l}\text { Maceri et } \\
\text { al. [26] }\end{array}$ & $\begin{array}{l}\text { 2nd Mandible } \\
\text { Premolar }\end{array}$ & 1.62 & 1.15 & 0.99 \\
\hline $\begin{array}{l}\text { Ren et al. } \\
{[10]}\end{array}$ & $\begin{array}{l}\text { 2nd Mandible } \\
\text { Premolar }\end{array}$ & 4.08 & 2.95 & 0.99 \\
\hline
\end{tabular}

\section{Results}

This section comprises the distribution of von Mises strain (VMST) and SED within the PDL, and von Mises stress (VMSR) and SED within the alveolar bone of the woPCE (without PCE) and WPCE (with PCE) models under $3 \mathrm{MPa}$ masticatory distributed load.

\section{1. von Mises strain (VMST) and strain energy density (SED) distribution within PDL}

The distribution of VMST and SED within PDL of the wOPCE and WPCE models under a $3 \mathrm{MPa}$ masticatory distributed load are shown in Fig. 3. These mechanical stimuli are shown along two paths on the PDL of the 2nd premolar in the woPCE and wPCE model. The 1st path (Path A) lies on the buccolingual section of the tooth ( $Y-Z$ plane), and the 2nd path (Path $B$ ) lies on the mesio-distal section of the tooth (X-Z plane) (Fig. 3a). It can be observed that in the most parts of both paths, the vMST and SED values within the PDL in the woPCE model are greater than those within the PDL in the WPCE model. The maximum and mean differences between the VMST of wOPCE and WPCE models along path A are $23 \%$, and $12 \%$, respectively, and along path $B$ are $27 \%$, and $13 \%$, respectively. Also, for SED, the maximum and mean differences between woPCE and WPCE models along path A are $59 \%$, and $31 \%$, respectively, and along path $B$ are $38 \%$, and $26 \%$, respectively. 


\section{2. von Mises stress (VMSR) and strain energy density (SED) within Alveolar Bone}

The VMSR and SED along two paths of alveolar bone in woPCE and WPCE models under a $3 \mathrm{MPa}$ masticatory distributed load can be seen in Fig. 4. These mechanical stimuli are shown along two paths within alveolar bone: the 1st path (Path A) lies on the bucco-lingual section of the bone ( $\mathrm{Y}$-Z plane) and the 2nd path (Path B) lies on the mesio-distal section of the bone (X-Z plane) (Fig. 4a). As can be observed in Fig. 4, the vMSR and SED values within the alveolar bone of the wPCE model are greater than those associated with the woPCE model. The maximum and mean differences between the vMSR in alveolar bone between the two models along path $A$ are $30 \%$, and $19 \%$, respectively, and along path $B$ are $19 \%$, and $17 \%$, respectively. Moreover, the maximum and mean differences between the SED within alveolar bone between the two models along path $A$ are $18 \%$, and $15 \%$, respectively, and along path $B$ are $28 \%$, and $23 \%$, respectively.

\section{Discussion}

In spite of recent investigations on the PDL-cementum enthesis [1,5], lack of research on its load transmitting function in the tooth-PDT-bone complex is evident in the current literature. This work aimed at investigating the impact of PDL-cementum enthesis on the mechanical stimuli transmission from cementum to PDL, and from PDL to alveolar bone within the tooth-PDT-bone complex, using the finite element method. Thus, two models were created: one model without PCE (WPCE), which consists of a 2nd premolar tooth and the alveolar bone (Fig. 1d); and the other model (WPCE), which was similar to the 1st one, but a thin layer with a thickness of $20 \mu \mathrm{m}$ was considered between the PDL and cementum, as PCE (Fig. 1e). The von Mises strain (vMST) and strain energy density (SED) within PDL of the 2nd premolar, also von Mises stress (VMSR) and SED within alveolar bone, in woPCE and in WPCE, under a 3 MPa distributed occluso-apical loading (see Fig. 1f) were found.

Strain energy density (SED) is a strong candidate for initiating bone remodeling process [27, 28], and also as a mechanical stimulus it results in cell response and migration in tendon and ligament [29-31]. So, it is expected that PDL tissue responds to the SED, which experiences during mastication [32], and also neighboring bone will be sensitive to it, as it can determine rate of bone remodeling $[33,28]$. The von Mises stress and strain are used as parameters that mitigate understanding of the overall stress and strain states in isotropic materials.

The differences observed between the displacement-force pattern of the 2nd premolar tooth in the wPCE model of this study (Fig. 2 and Table 2), with that of Brunski's experimental data [25], can be due to the inherent differences between the investigated teeth, i.e. the 1st maxillary molar in the later and the 2nd mandibular premolar in the former. Moreover, the differences between displacement-force pattern of this 
work with the finite element study of Ren et al. [10] can be due to the simplifications made in the geometry of tooth modeled in their study. Maceri and co-workers [26] performed the finite element analysis on an accurate 3D model of the 2 nd mandibular premolar, which was extracted from X-Ray images, and this may explain better similarity between displacement-force pattern of the 2nd premolar tooth of the WPCE model (Fig. 2 and Table 2), with that of Maceri et al. [26]. Taking into account the reasons for the differences and similarities, the discrepancy is considered to be reasonable and acceptable.

Results of this study showed that the investigated mechanical stimuli within PDL, i.e. VMST and SED under the $3 \mathrm{MPa}$ distributed occluso-apical load (see Fig. 1f) were different in WPCE from those of woPCE. Although identical geometries and the same set of material properties were used for finite element analysis, the wPCE model showed smaller values of vMST, as well as SED, within PDL than those associated with the woPCE model (see Fig. 3). The main hypothesized function for an enthesis is to reduce mechanical stimuli through applying them over a broader area, thereby increases fracture resistance and decreases failure risk [2, 34-36]. Ren et al. in a finite element study investigated the effect of cementum and cementum-dentin junction on the mechanical stimuli distribution within tooth-PDTbone complex [10]. They showed that cementum and CDJ have a significant impact on reducing PDL strains during mastication [10]. Results of the current study also showed that the alveolar bone experiences higher von-Mises stress and SED in wPCE model, compared with those of woPCE model (see Fig. 4), implying that in the WPCE model, the strain energy must be shifted from PDL to the alveolar bone. Considering that apparent density, and consequently strength of the bone are directly related to the extent of mechanical stimuli they experience $[33,28]$, thus one may conclude that wPCE results in a healthier bone, compared to that of WOPCE, in the long term.

Even though based on the currently available knowledge assigning a unique mechanical stimulus threshold for PDL resorption and damage under normal and non-pathological conditions is not possible yet, but it is known that increasing mechanical stimuli, specifically SED, within PDL, can cause some difficulties for the neighboring teeth structures, and may result in some diseases in the long term [37]. For instance, diastemata, a gap between teeth, which is associated with periodontal disease, is a result of mechanical stimuli rises and very large deformations within PDL [38, 37, 39]. Moreover, it was reported that increasing mechanical stimuli around alveolar crest region may lead to gingivitis, which causes microorganism entrance and infection [37]. Also, by increasing the age, the mechanical stimuli within PDL, especially in periapical region, increase and this may lead to the diseases in old individuals [37].

Healing and regeneration of a damaged PDL remains a significant challenge in clinical dentistry, which needs medical intervention to take place [40], but, on the other hand, bone has healing capability via continuous remodeling process $[41,42]$. It is speculated that by shifting the excess strain energy from PDL into the alveolar bone, PCE reduces the risk of PDL damage and diseases, and also increase neighboring bone's density and strength, through continuous bone remodeling process. Thus, the toothPDT-bone complex appears to be designed such that by reducing the mechanical stimuli within PDL, it decreases the risk of PDL damage under natural and non-pathological conditions [11]. 
It should be emphasized here that this study suffers from some limitations. First, the PCE was considered as a thin layer with homogenous material properties, and the gradual changes in its material properties was not taken into account. Second, uniform thicknesses were assigned to the PDT layers, and except for $\mathrm{PDL}$, linear elastic material properties were considered for other tissues, which may cause some errors. In spite of the fact that PDL is a viscoelastic material and shows time-dependent behavior, but since masticatory load was rapidly applied, time dependent properties were not considered here. Third, the lamina dura layer was not considered in this study. Forth, while the applied load to the 2nd premolar tooth was equivalent to $100 \mathrm{~N}$, the validation was performed for a range of $0-20 \mathrm{~N}$, due to non-availability of force-displacement data in the literature for upper values. Last, it should be noted that validation of the finite element models was performed through comparing results of this study with other experimental and FEM studies, and no experiments were done in this work.

\section{Conclusions}

Existence of PCE in the tooth-PDT-bone complex shifts the mechanical stimuli from PDL to its surrounding alveolar bone under masticatory load, and this can reduce the risk of PDL damage, as well as increase bone apparent density by amplifying strain energy density, and thus can construct a long lasting and low maintenance load bearing system. In regard to the biomechanical importance of PCE in the tooth-PDT-bone complex, even though results of this study seem promising, however further investigation is needed, for instance, by considering time dependent behavior and properties of both hard and soft tissues, to increase our understanding of enthesis functions in the load-bearing systems.

\section{Declarations}

\section{Acknowledgement}

The authors would like to thank Amirkabir University of Technology, Iran.

\section{Funding}

This research did not receive any specific grant from funding agencies in the public, commercial, or notfor-profit sectors.

\section{Conflicts of interest/Competing interests}

The authors declare that they have no competing interest.

\section{Availability of data and material (data transparency)}

The data supporting the results of this article are available.

Code availability (software application or custom code) 
No specific codes were written for this research.

\section{Authors' contributions}

All authors contributed to the study conception and design. Material preparation, data collection and analysis were performed by Hossein Jokar, Gholamreza Rouhi and Nabiollah Abolfathi. The first draft of the manuscript was written by Hossein Jokar and all authors commented on previous versions of the manuscript. All authors read and approved the final manuscript.

\section{References}

1. Ho, S. P., Marshall, S. J., Ryder, M. I., \& Marshall, G. W. (2007). The tooth attachment mechanism defined by structure, chemical composition and mechanical properties of collagen fibers in the periodontium. Biomaterials, 28(35), 5238-5245. doi:10.1016/j.biomaterials.2007.08.031.

2. Benjamin, M., Kumai, T., Milz, S., Boszczyk, B. M., Boszczyk, A. A., \& Ralphs, J. R. (2002). The skeletal attachment of tendons-tendon "entheses". Comparative biochemistry and physiology. Part $A$, Molecular \& integrative physiology, 133(4), 931-945. doi:10.1016/s1095-6433(02)00138-1.

3. BENJAMIN, M., \& McGONAGLE, D. (2001). The anatomical basis for disease localisation in seronegative spondyloarthropathy at entheses and related sites. Journal of Anatomy, 199(5), 503526. doi:10.1046/j.1469-7580.2001.19950503.x.

4. Roberts, W. E., Huja, S., \& Roberts, J. A. (2004). Bone modeling: biomechanics, molecular mechanisms, and clinical perspectives. Seminars in Orthodontics, 10(2), 123-161. doi:https://doi.org/10.1053/j.sodo.2004.01.003.

5. Lin, J., Jang, A., Kurylo, M., Hurng, J., Yang, F., Yang, L., et al. (2017). Periodontal ligament entheses and their adaptive role in the context of dentoalveolar joint function. Dental Materials, 33, doi:10.1016/j.dental.2017.03.007.

6. Chitsazan, A., Herzog, W., Rouhi, G., \& Abbasi, M. (2018). Alteration of Strain Distribution in Distal Tibia After Triple Arthrodesis: Experimental and Finite Element Investigations. Journal of Medical and Biological Engineering, 38(3), 469-481. doi:10.1007/s40846-017-0330-5.

7. Jokar, H., Rouhi, G., \& Abolfathi, N. (2020). The Effects of Splinting on the Initial Stability and Displacement Pattern of Periodontio-Integrated Dental Implants: A Finite Element Investigation. Journal of Medical and Biological Engineering. doi:10.1007/s40846-020-00544-5.

8. Rouhi, G., Tahani, M., Haghighi, B., \& Herzog, W. (2015). Prediction of Stress Shielding Around Orthopedic Screws: Time-Dependent Bone Remodeling Analysis Using Finite Element Approach. [journal article]. Journal of Medical and Biological Engineering, 35(4), 545-554. doi:10.1007/s40846015-0066-z.

9. Samsami, S., Saberi, S., Sadighi, S., \& Rouhi, G. (2015). Comparison of Three Fixation Methods for Femoral Neck Fracture in Young Adults: Experimental and Numerical Investigations. Journal of Medical and Biological Engineering, 35(5), 566-579. doi:10.1007/s40846-015-0085-9. 
10. Ren, L. M., Wang, W. X., Takao, Y., \& Chen, Z. X. (2010). Effects of cementum-dentine junction and cementum on the mechanical response of tooth supporting structure. J Dent, 38(11), 882-891. doi:10.1016/j.jdent.2010.07.013.

11. Nikolaus, A., Currey, J. D., Lindtner, T., Fleck, C., \& Zaslansky, P. (2017). Importance of the variable periodontal ligament geometry for whole tooth mechanical function: A validated numerical study. $J$ Mech Behav Biomed Mater, 67, 61-73. doi:10.1016/j.jmbbm.2016.11.020.

12. Ho, S. P., Goodis, H., Balooch, M., Nonomura, G., Marshall, S. J., \& Marshall, G. (2004). The effect of sample preparation technique on determination of structure and nanomechanical properties of human cementum hard tissue. Biomaterials, 25(19), 4847-4857. doi:10.1016/j.biomaterials.2003.11.047.

13. Kato, S., Nakagaki, H., Kunisaki, H., Sugihara, N., Noguchi, T., Ito, F., et al. (1992). The thickness of the sound and periodontally diseased human cementum. Arch Oral Biol, 37(8), 675-676. doi:10.1016/0003-9969(92)90132-r.

14. Natali, A. N., Pavan, P. G., \& Scarpa, C. (2004). Numerical analysis of tooth mobility: formulation of a non-linear constitutive law for the periodontal ligament. Dent Mater, 20(7), 623-629. doi:10.1016/j.dental.2003.08.003.

15. Pietrzak, G., Curnier, A., Botsis, J., Scherrer, S., Wiskott, A., \& Belser, U. (2002). A nonlinear elastic model of the periodontal ligament and its numerical calibration for the study of tooth mobility. Comput Methods Biomech Biomed Engin, 5(2), 91-100. doi:10.1080/10255840290032117.

16. Nishihira, M., Yamamoto, K., Sato, Y., Ishikawa, H., \& Natali, A. N. (2003). Mechanics of periodontal ligament. In A. N. Natali (Ed.), Dental Biomechanics. Bristol: Taylor and Francis Inc..

17. Pegoretti, A., Fambri, L., Zappini, G., \& Bianchetti, M. (2002). Finite element analysis of a glass fibre reinforced composite endodontic post. Biomaterials, 23(13), 2667-2682. doi:https://doi.org/10.1016/S0142-9612(01)00407-0.

18. Ho, S. P., Balooch, M., Goodis, H. E., Marshall, G. W., \& Marshall, S. J. (2004). Ultrastructure and nanomechanical properties of cementum dentin junction. J Biomed Mater Res A, 68(2), 343-351. doi:10.1002/jbm.a.20061.

19. Zargham, A., Geramy, A., \& Rouhi, G. (2016). Evaluation of long-term orthodontic tooth movement considering bone remodeling process and in the presence of alveolar bone loss using finite element method. Orthodontic Waves, 75(4), 85-96. doi:https://doi.org/10.1016/j.odw.2016.09.001.

20. Zhao, Y., Wang, W., Xin, H., Zang, S., Zhang, Z., \& Wu, Y. (2013). The remodeling of alveolar bone supporting the mandibular first molar with different levels of periodontal attachment. Med Biol Eng Comput, 51(9), 991-997. doi:10.1007/s11517-013-1078-x.

21. Ichim, I., Kieser, J., \& Swain, M. (2007). Functional significance of strain distribution in the human mandible under masticatory load: Numerical predictions. Arch Oral Biol, 52, 465-473. doi:10.1016/j.archoralbio.2006.10.020.

22. van Eijden, T. M. G. J. (1991). Three-dimensional analyses of human bite-force magnitude and moment. Arch Oral Biol, 36(7), 535-539. doi:https://doi.org/10.1016/0003-9969(91)90148-N. 
23. Asmussen, E., Peutzfeldt, A., \& Sahafi, A. (2005). Finite element analysis of stress in endodontically treated dowel restored teeth. J Prosthet Dent, 94, 321-329. doi:10.1016/j.prosdent.2005.07.003.

24. Joshi, S., Mukherjee, A., Kheur, M., \& Mehta, A. (2001). Mechanical performance of endodontically treated teeth. Finite Elements in Analysis and Design, 37(8), 587-601. doi:https://doi.org/10.1016/S0168-874X(00)00059-7.

25. Brunski, J. B. (1992). Biomechanical factors affecting the bone-dental implant interface. Clin Mater, 10(3), 153-201.

26. Maceri, F., Martignoni, M., \& Vairo, G. (2007). Mechanical behaviour of endodontic restorations with multiple prefabricated posts: a finite-element approach. J Biomech, 4O(11), 2386-2398. doi:10.1016/j.jbiomech.2006.11.018.

27. Rouhi, G., Epstein, M., \& Sudak, L. (2009). Modeling bone resorption using Mixture Theory with chemical reactions. Journal of Mechanics of Materials and Structures, 2 , doi:10.2140/jomms.2007.2.1141.

28. Vahdati, A., \& Rouhi, G. (2009). A model for mechanical adaptation of trabecular bone incorporating cellular accommodation and effects of microdamage and disuse. Mechanics Research Communications, 36(3), 284-293. doi:https://doi.org/10.1016/j.mechrescom.2008.10.004.

29. Devkota, A. C., Tsuzaki, M., Almekinders, L. C., Banes, A. J., \& Weinhold, P. S. (2007). Distributing a fixed amount of cyclic loading to tendon explants over longer periods induces greater cellular and mechanical responses. Journal of Orthopaedic Research, 25(8), 1078-1086. doi:10.1002/jor.20389.

30. Almekinders, L. C., Banes, A. J., \& Ballenger, C. A. (1993). Effects of repetitive motion on human fibroblasts. Medicine and science in sports and exercise, 25(5), 603-607.

31. Vermolen, F. J., \& Gefen, A. (2012). A semi-stochastic cell-based formalism to model the dynamics of migration of cells in colonies. Biomech Model Mechanobiol, 11(1), 183-195. doi:10.1007/s10237011-0302-6.

32. Carter, D. R., Fyhrie, D. P., \& Whalen, R. T. (1987). Trabecular bone density and loading history: Regulation of connective tissue biology by mechanical energy. J Biomech, 20(8), 785-794. doi:https://doi.org/10.1016/0021-9290(87)90058-3.

33. Huiskes, R., Ruimerman, R., van Lenthe, G. H., \& Janssen, J. D. (2000). Effects of mechanical forces on maintenance and adaptation of form in trabecular bone. Nature, 405(6787), 704-706. doi:10.1038/35015116.

34. Claudepierre, P., \& Voisin, M. C. (2005). The entheses: histology, pathology, and pathophysiology. Joint Bone Spine, 72(1), 32-37. doi:10.1016/j.jbspin.2004.02.010.

35. Genin, G. M., Kent, A., Birman, V., Wopenka, B., Pasteris, J. D., Marquez, P. J., et al. (2009). Functional Grading of Mineral and Collagen in the Attachment of Tendon to Bone. Biophysical Journal, 97(4), 976-985. doi:https://doi.org/10.1016/j.bpj.2009.05.043.

36. Ho, S. P., Kurylo, M. P., Fong, T. K., Lee, S. S., Wagner, H. D., Ryder, M. I., et al. (2010). The biomechanical characteristics of the bone-periodontal ligament-cementum complex. Biomaterials, 31(25), 6635-6646. doi:10.1016/j.biomaterials.2010.05.024. 
37. Cordes, V., Lüpke, M., Gardemin, M., Seifert, H., \& Staszyk, C. (2012). Periodontal biomechanics: Finite element simulations of closing stroke and power stroke in equine cheek teeth. BMC veterinary research, 8, 60. doi:10.1186/1746-6148-8-60.

38. Staszyk, C., \& Gasse, H. (2005). Distinct fibro-vascular arrangements in the periodontal ligament of the horse. Arch Oral Biol, 50, 439-447. doi:10.1016/j.archoralbio.2004.10.001.

39. Bourauel, C., Freudenreich, D., Vollmer, D., Kobe, D., Drescher, D., \& Jäger, A. (1999). Simulation of orthodontic tooth movements. A comparison of numerical models. J Orofac Orthop, 60(2), 136-151. doi:10.1007/bf01298963.

40. Andreasen, J. O. (2012). Pulp and periodontal tissue repair - regeneration or tissue metaplasia after dental trauma. A review. Dent Traumatol, 28(1), 19-24. doi:10.1111/j.1600-9657.2011.01058.x.

41. Little, N., Rogers, B., \& Flannery, M. (2011). Bone formation, remodelling and healing. Surgery (Oxford), 29(4), 141-145. doi:https://doi.org/10.1016/j.mpsur.2011.01.002.

42. Rouhi, G. (2012). Biomechanics of Osteoporosis: The Importance of Bone Resorption and Remodeling Processes: INTECH Open Access Publisher.

\section{Figures}


a
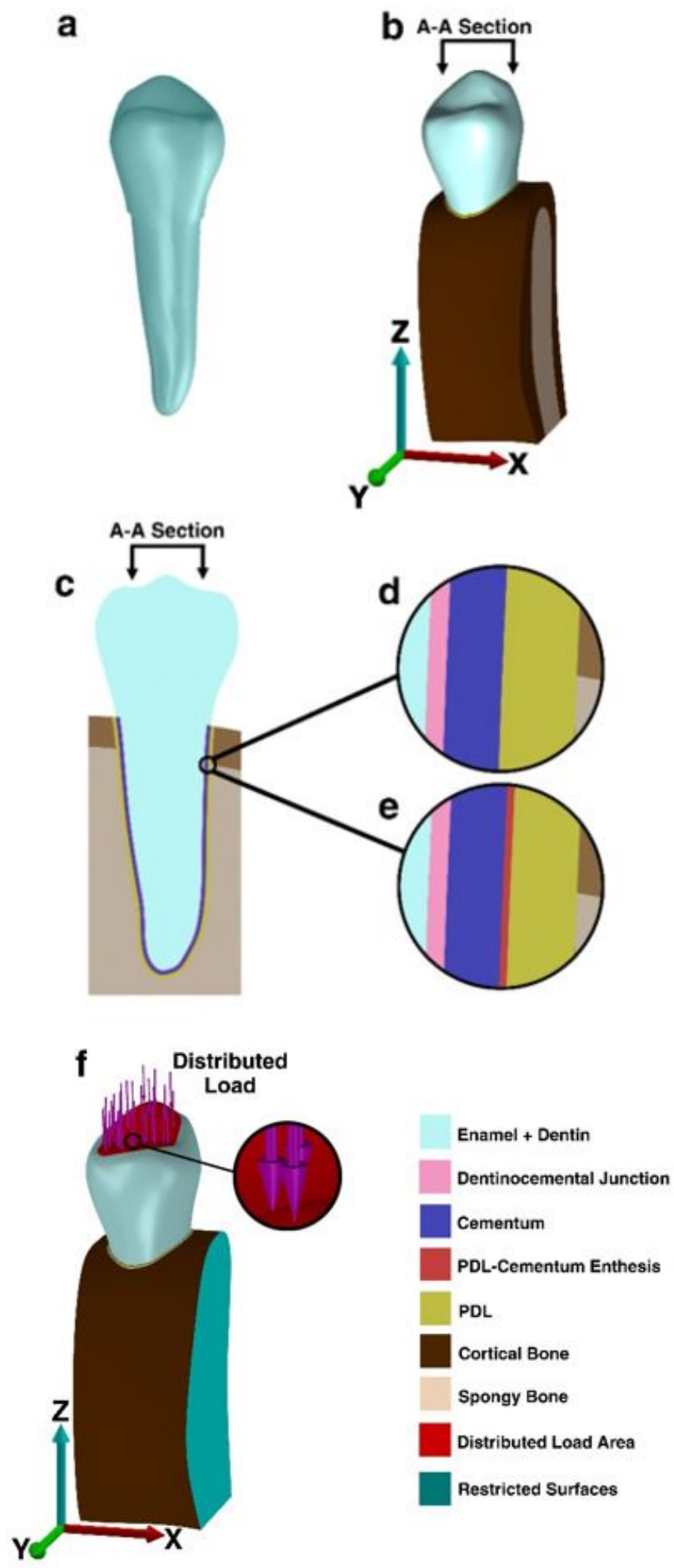

\section{Figure 1}

Three dimensional (3D) finite element models: (a) The 2nd premolar tooth model; (b) The 2nd premolar tooth and alveolar bone; and (c) Cross-section of the 2nd premolar tooth, the cortical and spongy parts of alveolar bone, and PDT; (d) Three layers of the PDT in the woPCE model (PDL, Cementum, and CDJ); (e) Four layers of the PDT in the wPCE model (PDL, PCE, Cementum, and CDJ); (f) Boundary conditions 
showing the distributed load region over the crown of the 2 nd premolar tooth, and the distal and mesial faces of alveolar bone constrained in all directions.

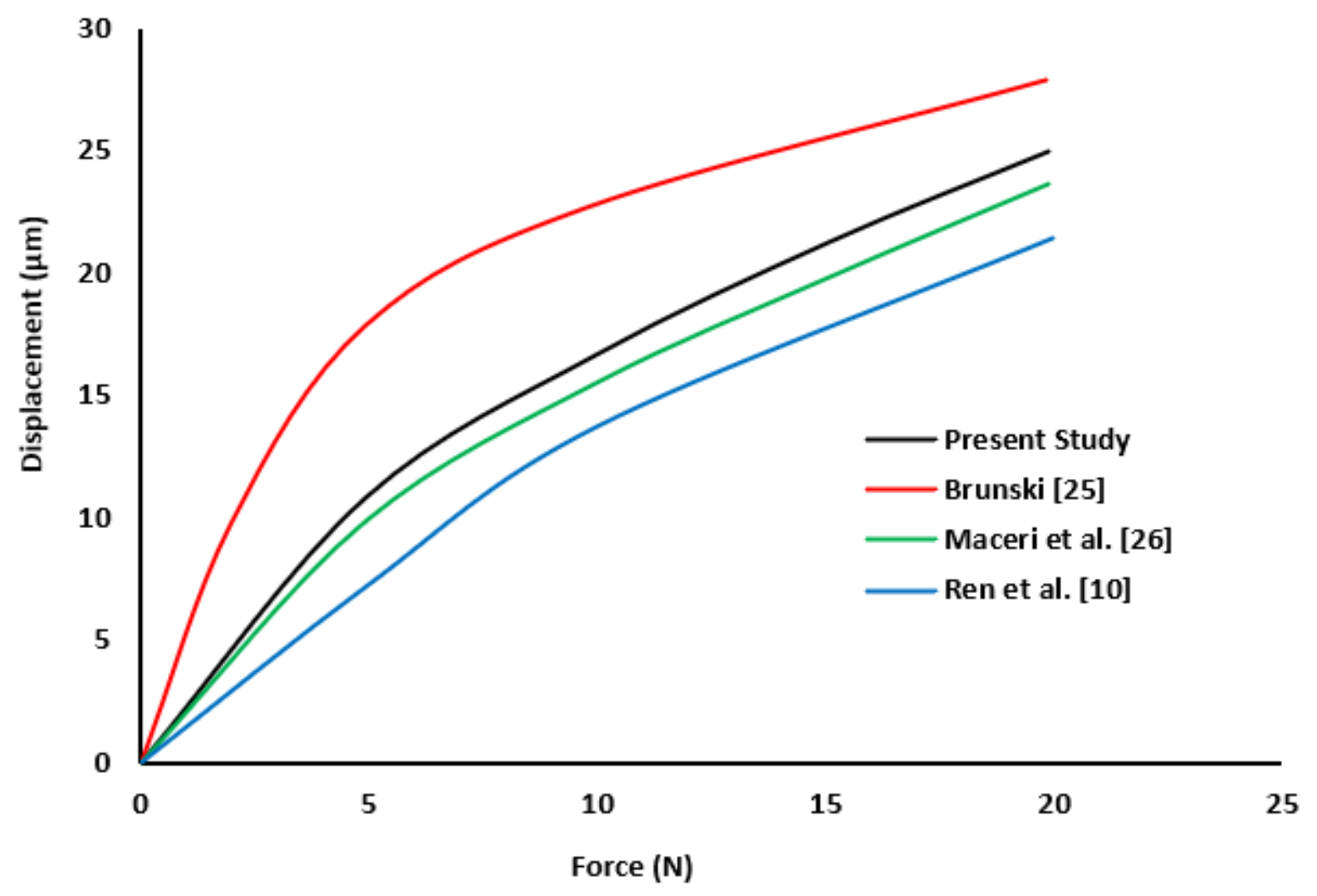

Figure 2

Displacement-force pattern of 2nd premolar tooth in the WPCE model under occluso-apical distributed masticatory load (Fig. 1f) resulted from the FE model of this study, and results of other investigations $[26,10,25]$. 


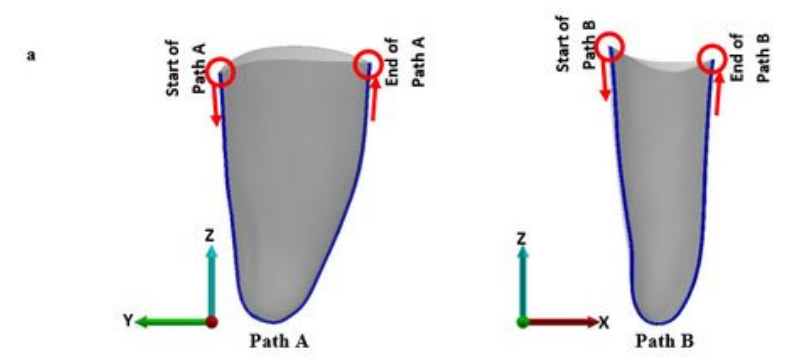

b
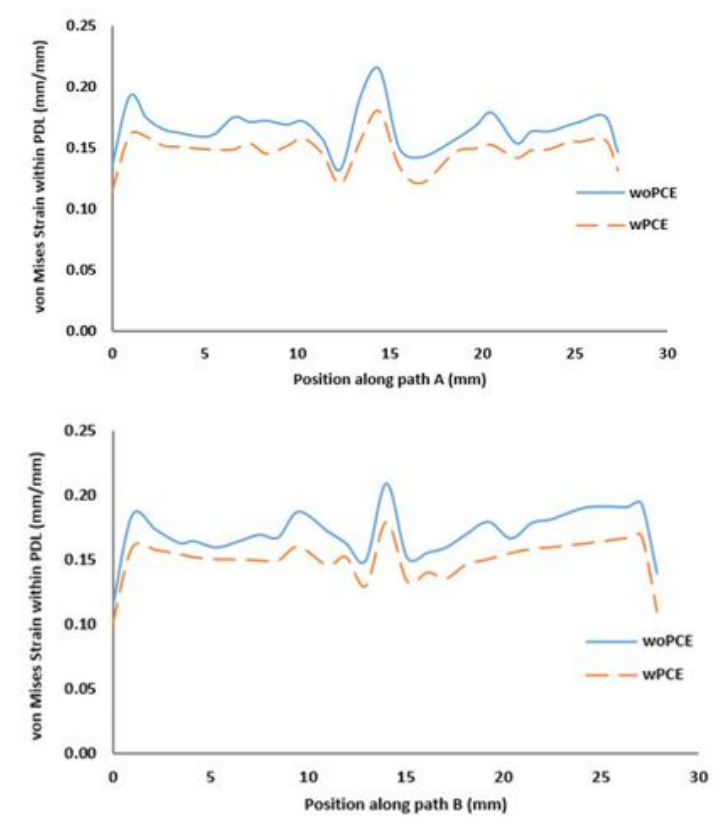

d
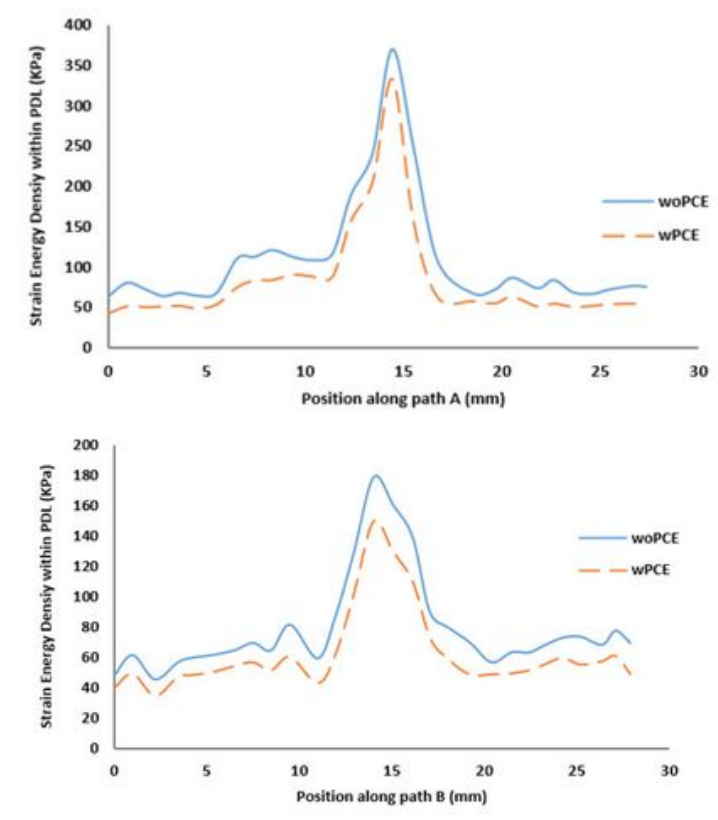

\section{Figure 3}

von Mises strain (vMST) and strain energy density (SED) distribution within PDL in the wPCE (with PCE) and woPCE (without PCE) models, along Path A and Path B caused by a 3 MPa masticatory distributed load (Fig. 1f) : (a) Path A (on the bucco-lingual section of tooth), and path B (on the mesio-distal section of tooth) on the PDL of the 2nd premolar tooth; (b) vMST within PDL of the wPCE and woPCE models along path $A$; (c) vMST within PDL of the wPCE and woPCE models along path B; (d) SED within PDL of 
the wPCE and woPCE models along path A; and (e) SED within PDL of the WPCE and WoPCE models along path $\mathrm{B}$.

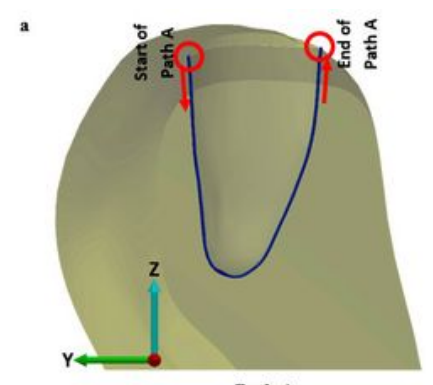

Path A

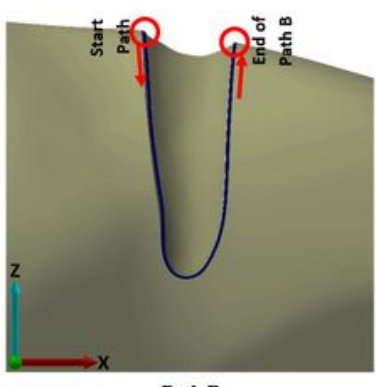

Path B

b
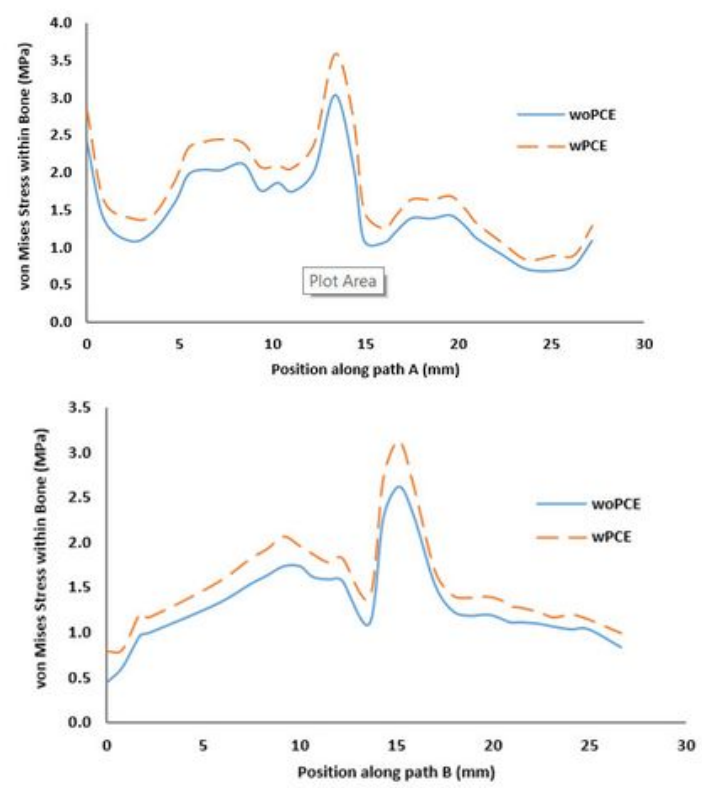

d
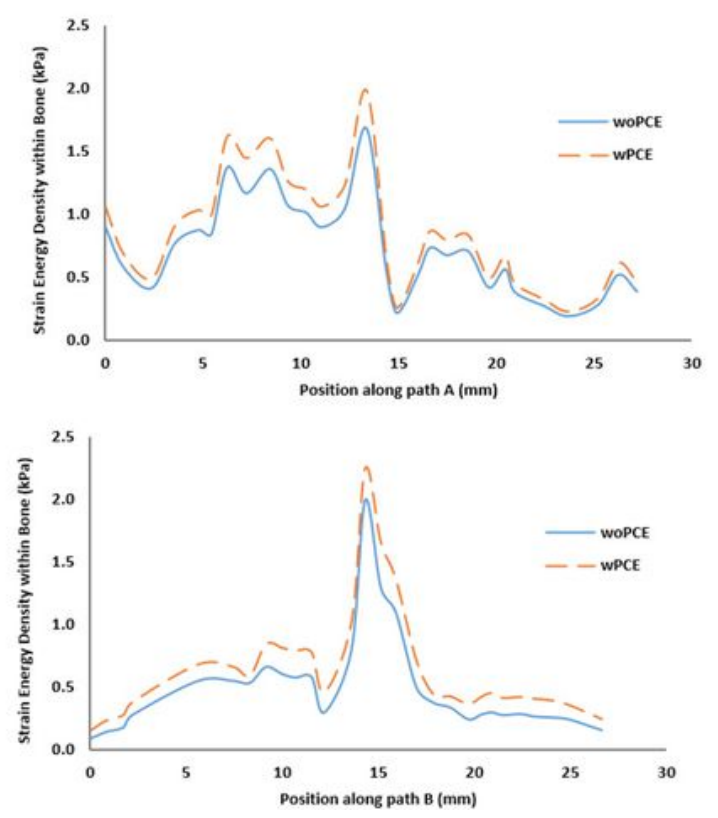

Figure 4

von Mises strain (VMST) and strain energy density (SED) distribution within alveolar bone in the wPCE (with PCE) and woPCE (without PCE) models, along Path A and Path B, caused by a 3 MPa masticatory distributed load (Fig. 1f) : (a) Path A (on the bucco-lingual section), and path B (on the mesio-distal 
section) on the alveolar bone; (b) VMSR within alveolar bone in the WPCE and wOPCE models along path $A ;$; (c) VMSR within alveolar bone in the WPCE and wOPCE models along path B; (d) SED within alveolar bone in the wPCE and wOPCE models along path A; and (e) SED within alveolar bone in the WPCE and woPCE models along path $B$. 